\title{
Virtual cues and functional mobility of people with Parkinson's disease: A single-subject pilot study
}

\author{
Tatiana A. Kaminsky, MS, OTR; ${ }^{1-2 *}$ Brian J. Dudgeon, PhD, OTR; ${ }^{3}$ Felix F. Billingsley, PhD; ${ }^{4}$ Pamela H. \\ Mitchell, PhD, CNRN, FAAN; ${ }^{1}$ Suzanne J. Weghorst, MS $^{5}$ \\ ${ }^{1}$ School of Nursing, University of Washington, Seattle, WA; ${ }^{2}$ Department of Occupational Therapy, University of Puget \\ Sound, Tacoma, WA; ${ }^{3}$ Department of Rehabilitation Medicine, ${ }^{4}$ College of Education, ${ }^{5}$ Human Interface Technology \\ Laboratory, University of Washington, Seattle, WA
}

\begin{abstract}
In this study, adults with Parkinson's disease (PD) used virtual cueing spectacles (VCS) mimicking kinesia paradoxa in home and community settings to assess the impact on mobility and participation. We used an ABA single-subject design with repeated measures. Six adults with PD, akinesia, and stage III or IV Hoehn and Yahr rating scale status used VCS in their homes and communities for a week or more. Our main outcome measures included participant counts of losses of balance and freezes, pre-/postintervention completion of the Parkinson's Disease Questionnaire-39, observation of baseline and intervention gait, and an interview regarding user satisfaction with VCS. We also assessed participants' preuse baseline and return to baseline. Use of VCS decreased length of freezes as well as number of freezes for some participants. All participants expressed satisfaction with VCS. VCS shows promise in simulating kinesia paradoxa to improve the gait of some adults with PD in the home and community.
\end{abstract}

Key words: accidental falls, activities of daily living, assistive technology, community participation, cues, equipment and supplies, festinating gait, gait apraxia, kinesia paradoxa, mobility limitation.

\section{INTRODUCTION}

Parkinson's disease (PD) is a chronic and degenerative neurological disorder that leads to performance changes that affect an individual's ability to stay active.
PD results in severe disability in approximately 1 percent of people over the age of 60 [1-2], including veterans. The Department of Veterans Affairs treats an estimated 40,000 veterans with PD each year [3]. Akinesia, which typically occurs about 10 years after onset [4-5], is one of the most debilitating symptoms of PD. Studies report prevalence of akinesia ranging from 32 [4] to 60 percent [6] among people with PD.

Akinesia is noted most prominently in the gait pattern of the person with PD. Akinetic gait is seen as a series of shuffling steps with reduced step length [7-8] and sometimes includes moments when movement ceases completely. These moments are referred to as "frozen gait,"

\footnotetext{
Abbreviations: $\mathrm{ADL}=$ activities of daily living, $\mathrm{IADL}=$ instrumental ADL, LOB = loss of balance, MMSE = Mini-Mental Status Examination, $\mathrm{PD}=$ Parkinson's disease, $\mathrm{PDQ}-39=$ Parkinson's Disease Questionnaire-39, VCS = virtual cueing spectacles.

*Address all correspondence to Tatiana A. Kaminsky, MS, OTR; Department of Rehabilitation Medicine Box 356490, University of Washington, Seattle, WA 98195; 206-5985412; fax: 206-685-3244. Email: tak@u.washington.edu Request reprints from Brian J. Dudgeon, PhD, OTR; Department of Rehabilitation Medicine, P.O. Box 356490, University of Washington, Seattle, WA 98195; 206-598-5412; fax: 206685-3244. Email: dudgeonb@u.washington.edu
}

DOI: 10.1682/JRRD.2006.09.0109 
which is generally described as feeling as if the feet are glued to the floor $[4,6,8]$. Freezing is most common in initiating gait, turning, and walking through doorways or narrow spaces [2,4-6,9-10]. In addition to physical location, freezing is influenced by the person's emotions, appearing more frequently in times of distress [9] or when the person is attempting complex tasks, such as doing two things at once [10]. Freezing gait also increases the risk for falls [12,5,9]. Gray and Hildebrand examined 118 people with PD and found that freezing ". . . is closely linked to reports of falling. Of patients with occasional or frequent episodes of freezing, 80 percent experienced falls” [1, p. 224]. Greater depression and lesser quality of life [11] are also found among those who have a history of falls, gait disturbances, and akinesia and rigidity [12], especially if they subsequently experience further decline in physical mobility, emotional reactions, pain, and social isolation [13].

\section{Sensory Cueing to Overcome Akinesia}

People with akinesia due to PD often continue to experience gait impairment even with current medical treatments $[8,14]$. As a result, nonpharmacological approaches are needed for akinesia, including the use of external sensory cues to help the person initiate or maintain movement. People with PD use a wide variety of techniques to overcome akinesia, including being pushed, marching to a cadence, rocking the body, walking over objects, and walking to music, but over time these strategies lose effectiveness [15]. Little research has examined the effects of auditory or tactile cueing strategies. Research on the use of auditory cues, such as a metronome, has found that these cues can positively affect gait initiation and maintenance [16], as well as gait cadence [17], but when used alone or in combination with visual cues results are mixed [18-19]. Most of these studies have been conducted in laboratory environments. The effects of auditory cues in the community and their long-term effectiveness have not been examined.

In contrast to auditory cueing, more research has been conducted on the use of visual cueing to overcome akinesia. External visual cues are tried most often to create a phenomenon known as kinesia paradoxa that has been well documented in the literature [2,7-9,19-20]. People with akinesia who demonstrate kinesia paradoxa are observed to walk with visual cue patterns, such as over obstacles in their path or up stairs, with significantly reduced shuffling and freezing gait [2,7,9-10,19-20].
Until now, researchers have found that visual cues must be affixed to or projected on a surface. As a result, nearly all the studies involving kinesia paradoxa have been conducted in the laboratory environment. Dietz et al. tested the use of a modified walking stick that provided horizontal visual cues as a way of overcoming this limitation [9]. While participants reported effective community use, the researchers did not observe this because the study was conducted within a laboratory setting. Kompoliti et al. tested the use of a modified inverted stick as a visual cue, as well as a projected laser beam stick to overcome gait freezing in a laboratory setting [21]. They found inconsistent results across patients with PD and suggested that only some might benefit from long-term use with supervised training.

Some researchers have examined the lasting effects of visual cues on the gait of people with PD. In a case study by Sidaway et al., the gait speed, step length, and lower-limb kinematics of a woman with PD were measured at baseline, with visual cues, and after removal of cues on a laboratory walkway [22]. The woman studied continued to show improvement in gait 1 month after the visual cues were no longer used. Changes in community mobility were not reported. Most other researchers have reported that when the cues are removed, shuffling steps and frozen gait reappear [10]. Because of the need for a physical surface on which to place visual cues and because most evidence indicates that the effects of kinesia paradoxa do not last after the removal of visual cues, the functional usefulness of this phenomenon to the lives of people with akinesia has been limited.

\section{Virtual Cueing Technology}

Recently, the Human Interface Technology Laboratory at the University of Washington (Seattle, Washington), in conjunction with HMD Therapeutics (San Anselmo, California), designed a pair of spectacles that provides visual cues in a format that can be taken into many environments, including the community [23-24]. These spectacles consist of a light-emitting diode display on one side that generates a series of horizontal lines that are reflected off a lens into the wearer's eye. Thus, when the wearer is looking at the ground, the lines appear to be on the walking surface in front of the user. The spectacles can be used to simulate the use of external visual markers in laboratory, home, and community environments. This format, described by Riess as a "central field cueing device" [24], has been incorporated into testable prototypes by Enhanced Vision 
Systems (Huntington Beach, California) under a licensing agreement from HMD Therapeutics. In this report we refer to these prototypes as "virtual cueing spectacles" (VCS).

Functional impact from use of the device has begun to be explored. Prothero studied gait effects of an earlier VCS prototype [25]. A 6.4 percent improvement in stride length was found when two adults with PD used the VCS in a laboratory setting. A more extensive laboratory-based study was conducted by Weghorst and Kaminsky, who assessed 52 participants walking a 15-foot path naturally (with no visual cues) with physical markers and with the VCS [26]. A significantly longer step length was seen when participants used physical cues than when they walked naturally, supporting the presence of kinesia paradoxa. Subsequently, these participants walked with a significantly longer step length when they used the VCS than when they walked naturally, demonstrating that the VCS improved participants' gait in this laboratory setting.

A need still exists for assessing the functional impact of VCS in natural settings and the community. The purpose of our study was to assess the impact of the VCS on the functional mobility of people with akinesia due to PD in their home and community environments. Our specific research questions were (1) Do the VCS decrease the number of losses of balance (LOBs) or freezing episodes or the length of freezing episodes? (2) What impact does the use of VCS have on active versus sedentary lifestyles? and (3) Do users find the VCS helpful in their home and community environments?

\section{METHODS}

\section{Participants}

Six people with idiopathic PD (Table 1) participated in a repeated measures ABA (baseline-intervention-baseline) single-subject research design following procedures approved by an institutional review board. Each had changes in gait, with difficulty reported in household and community mobility. Each participant was recruited from a pool of people who had participated in a laboratory-based study of the VCS and expressed interest in future studies.

The PD stage of all participants was determined through examination and interview by the principal investigator (TAK) and subsequent use of the five-stage Hoehn and Yahr rating scale [27] that demonstrates good reliability among experienced and inexperienced raters [28]. Inclusion criteria were a Stage III or IV rating on the Hoehn and Yahr rating scales and a Mini-Mental Status Examination (MMSE) [29] score of at least 27 out of the possible 30. Hoehn and Yahr Stage III is characterized by unsteadiness when turning or when pushed with eyes closed. People in Stage III are still able to complete self-care tasks independently. Persons in Stage IV can walk and stand unassisted, but cannot independently complete all self-care tasks. The MMSE minimum score assured cognitive functioning sufficient for use of the VCS and completion of daily record-keeping. Participants were excluded if they used a walker for ambulation, had a gait disorder not attributed to $\mathrm{PD}$, or a visual impairment that limited their ability to see their feet or the virtual cues.

Table 1.

Participant characteristics.

\begin{tabular}{|c|c|c|c|c|c|c|}
\hline Participant & Age & Sex & $\begin{array}{l}\text { Kinesia Paradoxa } \\
\text { in Laboratory }\end{array}$ & $\begin{array}{l}\text { Years with } \\
\text { PD Dx, Rx }\end{array}$ & $\begin{array}{c}\text { Hoehn \& Yahr } \\
\text { Rating }\end{array}$ & Residence \\
\hline $\mathrm{I}$ & 72 & Female & Yes & 8, Medication & III & Retirement home \\
\hline III & 76 & Male & No & 18, Medication & III & $\begin{array}{l}\text { Own home, with wife \& } \\
\text { daughter }\end{array}$ \\
\hline $\mathrm{V}$ & 70 & Male & No & 10, Medication & IV & $\begin{array}{l}\text { Own home, with wife \& } \\
\text { daughter }\end{array}$ \\
\hline VI & 50 & Female & No & $\begin{array}{l}\text { 16, Medication \& } \\
\text { Pallidotomy left side }\end{array}$ & III & $\begin{array}{l}\text { Own home, with son \& his } \\
\text { family }\end{array}$ \\
\hline
\end{tabular}




\section{Intervention Design and Device}

In this ABA design, baseline phases were 7 to 10 days long, followed by planned use of the VCS in the home and community environments for 10 days and subsequent follow-up without use of the VCS for 1 to 4 days. Enhanced Vision Systems manufactured a prototype of the VCS that was available for each participant (Figure 1). The VCS were programmed for participants so that when they looked directly at their feet, they would see stationary horizontal lines of light, like the rungs of a ladder, on the floor in front of them. As they raised their heads to look slightly ahead of their feet, the horizontal lines began to scroll, appearing to come toward them as they walked. This virtual line provided a "step over" target as they walked forward. When they looked directly forward, the light display would turn itself off and would reappear if participants' heads tilted downward toward their feet. The study glasses could be worn over the participant's prescription eyeglasses and use of bifocals or trifocals did not appear to interfere with the use of the VCS.

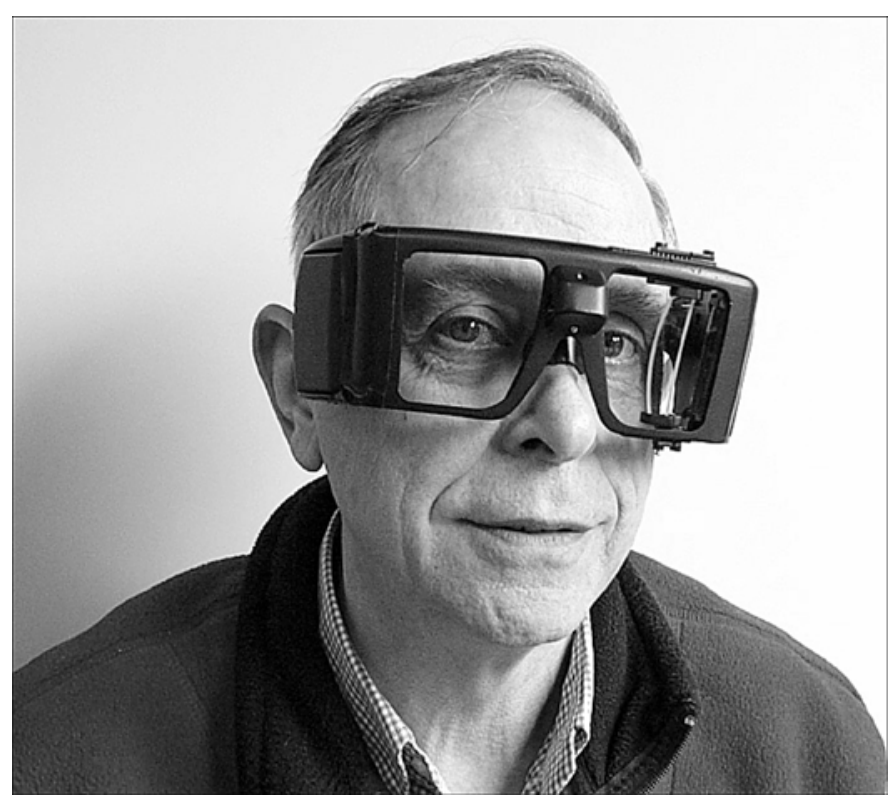

Figure 1.

Prototype of virtual cueing spectacles (VCS) used in study. More streamlined version is planned for commercial release by Enhanced Vision Systems (Huntington Beach, California). VCS contain power source (cell phone battery) on right side. Left side has set of orange lights, oriented vertically, located near front of glasses. Thin lens in middle of opening for left eye reflects light from left light source into user's eye. Small buttons on left side of glasses used to set speed at which lights scroll and head-tilt points that trigger lights.

\section{Measures and Data Collection Procedures}

Participants were trained to monitor and log LOBs, freezing episodes, and participation in activity on a daily basis. The principal investigator observed participants' gait once in baseline and once with VCS use. In addition, participants were telephoned every 2 to 3 days to monitor data collection procedures. Pre- and postintervention scores on the Parkinson's Disease Questionnaire-39 (PDQ-39) [30] were used as outcome measures as well. Before we began the intervention, we trained participants in the settings, use, and care of the VCS.

\section{Losses of Balance and Freezing}

LOBs were events during which participants had difficulty maintaining their upright position but were able to regain their balance before falling. Falls were defined as times when hands or knees unintentionally came into contact with the floor or other lower surface. Falls were counted as LOBs. Freezing episodes were occasions when participants were unable to initiate or sustain mobility. We created training videotapes using nonstudy participants who demonstrated freezing episodes and LOBs. Participants viewed these videotapes and scored the number of freezing episodes and LOBs. Training continued until participants and the investigator reached 95 percent or better agreement about definitions of LOBs and freezing episodes. A real-time estimate of freezes and LOBs was conducted as part of the study. This is described in the "Methods of Analysis" section.

Participants used daily data sheets that showed the period of time between getting out of bed in the morning until going to sleep at night to record LOBs or falls and freezing episodes. Awakenings in the night were disregarded in terms of data collection. Participants recorded the numbers of LOBs or falls and freezing episodes and noted where these events occurred. Wrist counters were provided to the participants to facilitate record keeping.

\section{Activity Level}

Participants marked the types of daily activities they engaged in on a 38-item checklist. Activities on the checklist included personal activities of daily living (ADL), instrumental ADL (IADL), and leisure and social activities.

\section{Parkinson's Disease Questionnaire and Exit Interview}

The PDQ-39 is a 39-item questionnaire that assesses people's perceptions about the impact of PD on aspects of 
their lives (i.e., mobility, ADL, emotional well-being, stigma, social support, cognition, communication, and bodily discomfort) [30]. Each question rates how often during the past month (e.g., from never [0] to always [4]) the person experienced an emotion or symptom of PD. Each subsection receives a separate score scaled from 0 to 100. A higher score indicates a higher perception of difficulty due to PD. Following training on data collection, each participant completed the PDQ-39 and began the baseline phase. After the study was concluded, participants again completed the PDQ-39 and were interviewed by the principal investigator about the usefulness of the VCS and other observations of their own functioning.

\section{Reliability Checks}

Interobserver agreement was assessed one time each during the baseline and intervention phases through observation sessions. At these times, participants completed an activity in the home or community, such as a trip to the grocery store or meal preparation. The investigator observed the participant's gait characteristics and counted the number of LOBs and freezing episodes. These data were compared with the participant's ratings for the same period. Interobserver agreement between the participant and the investigator was assessed one time each during the baseline and intervention phases. Agreement ranged from 85.7 to 100 percent during both phases.

Daily data collection procedures and use of the VCS were also monitored through telephone calls every 2 to 3 days. The investigator answered questions about data sheets as well as participants' use of the VCS. If a second party was present, such as a spouse or caregiver, a procedural confirmation was carried out regarding data collection and VCS use.

\section{Methods of Analysis}

Daily freezing episodes, LOBs, and activity participation data for each participant were collected from the data sheets. The investigator determined the amount of time a participant spent frozen by noting and timing the participant's freezing episodes in observed home/ community sessions. The average amount of time spent in a freeze during the observation session was then calculated for each participant by dividing the total amount of time the participant spent in a frozen state by the total number of freezes observed by the researcher.
We graphed and visually analyzed all data by comparing baseline data to intervention data, with an emphasis on high and low data points and trends within phases (Figures 2-5). Trend lines derived by split-half analysis [31] were used to assist in visual analysis, but are not displayed because of high variability of data from many participants. Pre- and postintervention scores on the PDQ-39 and selected comments from participants during the exit interview regarding perceived helpfulness of VCS are reported.

\section{RESULTS}

\section{Freezing Episodes and Losses of Balance}

No participant appeared to have a worsened gait with use of the VCS as measured by freezing episodes or LOBs. One participant, Participant V (Figures 3(b) and 5(b)), had clear and immediate reductions in both freezing episodes and LOBs when he began using the VCS. These improvements reversed immediately when he stopped using the VCS. Use of the device clearly affected the numbers of both freezing episodes and LOBs experienced by this participant, but variability among the other five participants precluded identification of clear patterns of effect on either feature of walking.

Several participants showed reduced numbers of freezing episodes while using the VCS. Participant I had a change in her Parkinson's medications during the baseline period that may have decreased her number of freezing episodes each day. When she began using the VCS, she maintained this decline in freezing, except for one day when she was ill and had an increase in freezing episodes. When she stopped using the VCS, she had an immediate increase in freezing episodes (Figure 2(a)). For Participant II, after he retrained with the VCS on day 12, his number of freezing episodes dropped sharply and remained relatively level for the remaining intervention period. His number of freezing episodes increased again when he stopped using the VCS (Figure 2(b)). The number of freezing episodes decreased for Participant III during baseline, stayed low while he used the VCS, and then began rising when he stopped using the VCS. He had the fewest episodes of freezing during the intervention period and the highest during the baseline periods. Participant IV experienced fewer freezing episodes in the intervention period than during the initial baseline period (Figure 3(a)). Participant VI showed a decrease in the number of freezing 
(a)
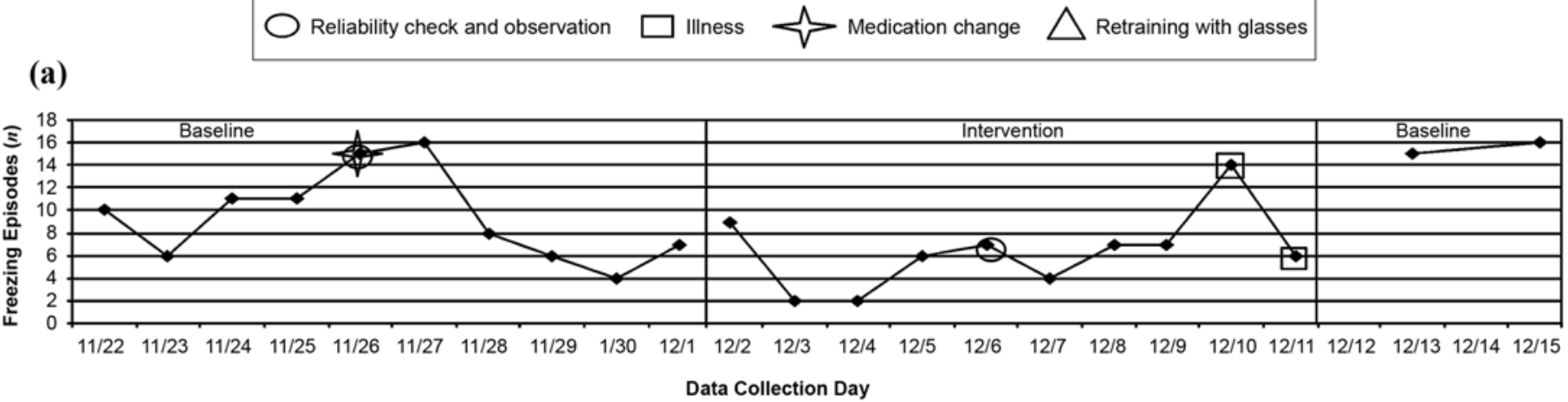

(b)

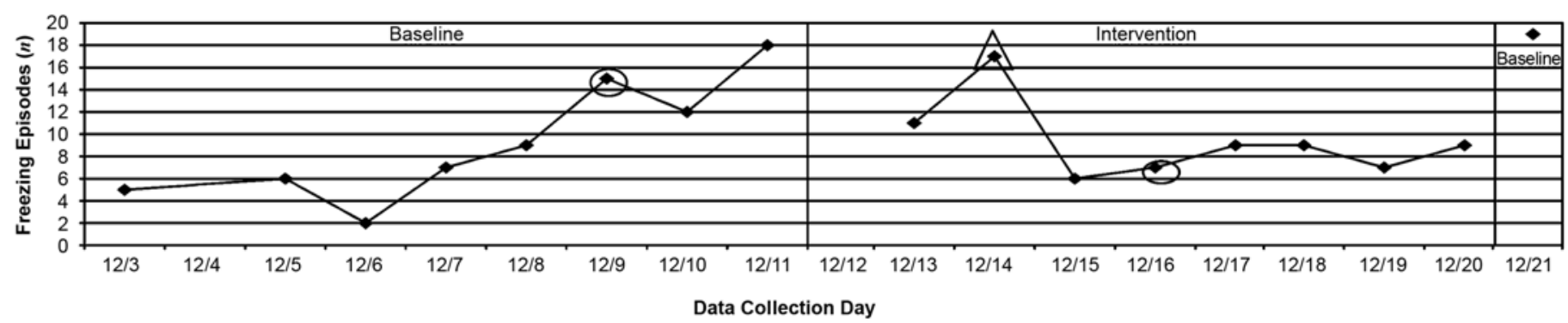

(c)

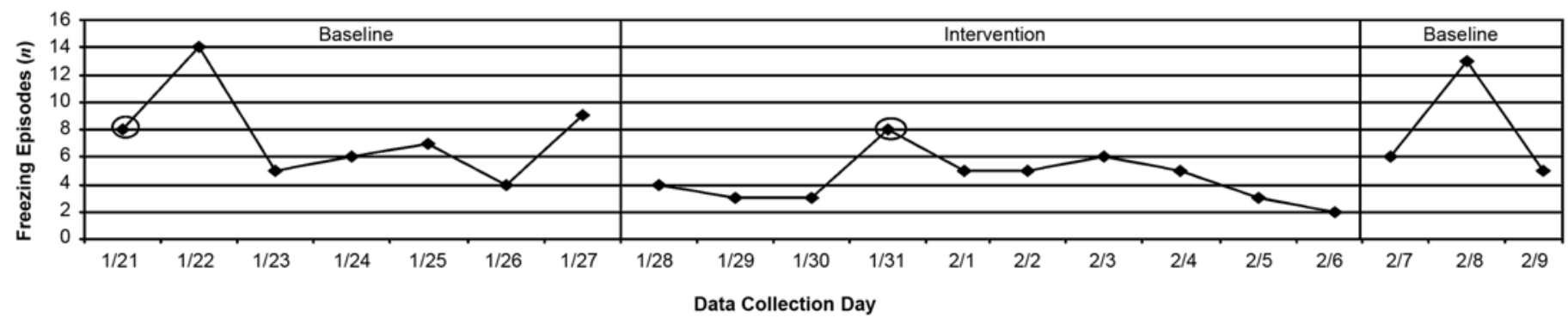

Figure 2.

Daily recordings of freezing episodes for (a) Participant I, (b) Participant II, and (c) Participant III.

episodes after she began using the VCS (Figure 3(c)). Even with a medication change, use of the VCS appeared to decrease her number of freezing episodes.

For five participants, the average amount of time spent in a freezing episode during observed sessions in the intervention period was shorter than during the baseline period (Figure 6). Participant IV did not experience a freezing episode during either the baseline or intervention observations, so the impact of the VCS on length of his freezing episodes could not be determined.

The impact on LOB frequency could not be clearly determined for most participants. For Participants I, II, IV, and VI, the data were highly variable (Figures 4 and 5).
As a result, we could not determine clear patterns of impact from use of the VCS on LOB. Participant III had only one LOB during the course of the study (Figure 4(c)), so we could not identify the impact of the VCS on LOB for this participant.

\section{Gait Change Characteristics}

All six participants were observed to have qualitative improvement in their gait when they used the VCS, including larger step length (Participants I, V, and VI), decreased rigidity during mobility (Participants I, II, IV, and VI), increased arm swing (Participants II and VI), and increased ground clearance with decreased shuffling (Participants III, 


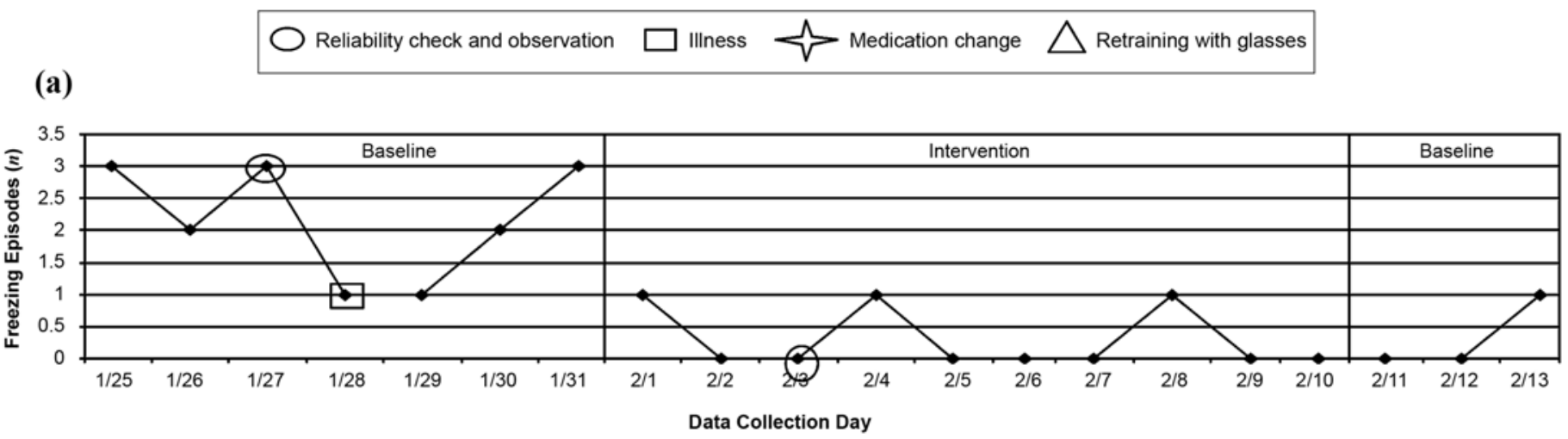

(b)

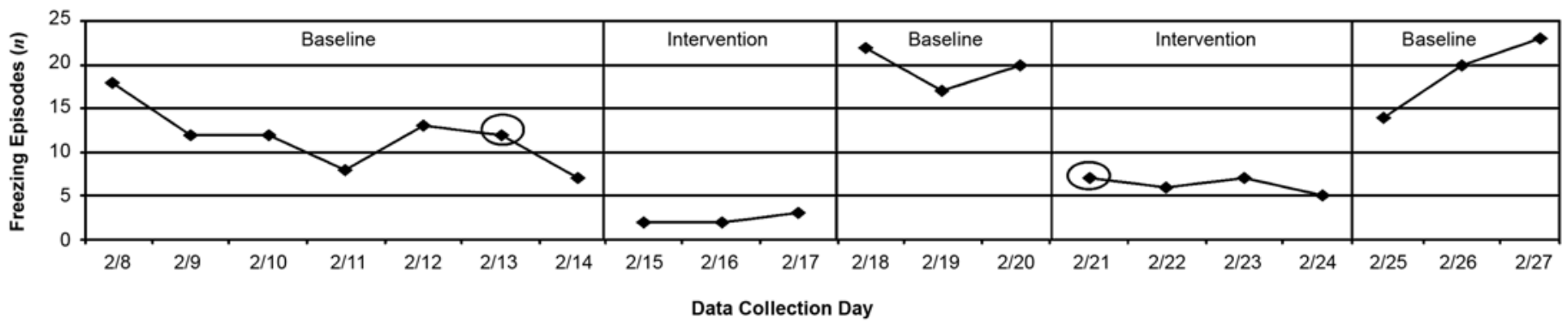

(c)

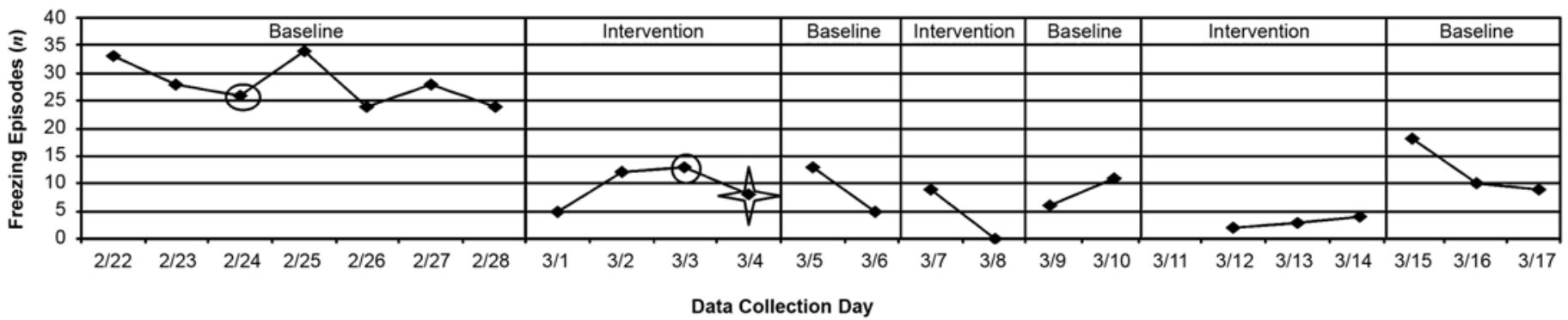

Figure 3.

Daily recordings of freezing episodes for (a) Participant IV, (b) Participant V, and (c) Participant VI.

IV, V, and VI). Improvements in dyskinetic gait were also noted with Participants II and VI. No change was noted in tremor in any of the participants.

\section{Activity Participation}

We monitored activity engagement daily to assess the impact that VCS use had on activity participation. Activity data for five of the participants (I, II, IV, V, and VI) were relatively level, which did not clearly demonstrate either a positive or negative effect of VCS use on activity participation. Participant III showed an increase in inside activity during the intervention period, but his outside activity data showed high variability, with no clear pattern based on use of VCS. His total activity slightly increased during the intervention period, but overall the VCS did not have a clear effect on Participant III's activity participation. We do not show the activity data graphs here because we did not see a clear effect of the VCS on activity participation.

\section{Participants' Perception of Efficacy}

Five of the participants (I, III, IV, V, and VI) had improvements in perception of difficulty scores in the mobility category of the PDQ-39 (Table 2). Other score 
(a)
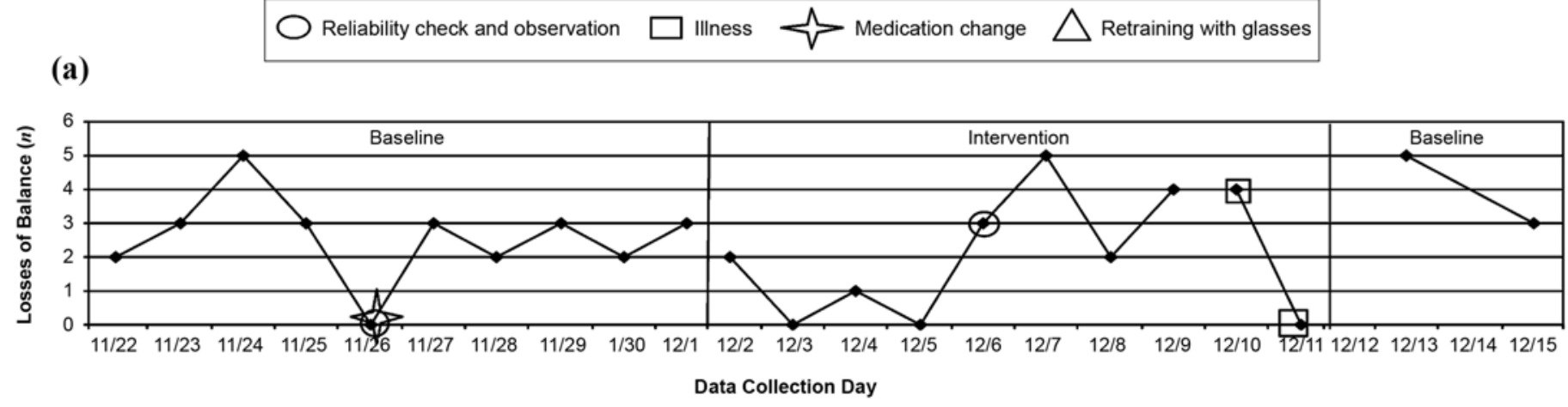

(b)

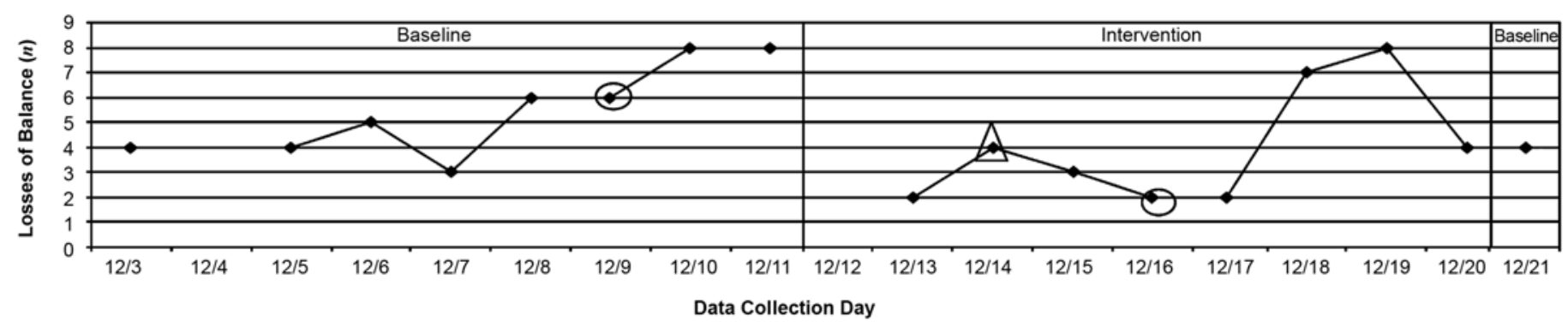

(c)

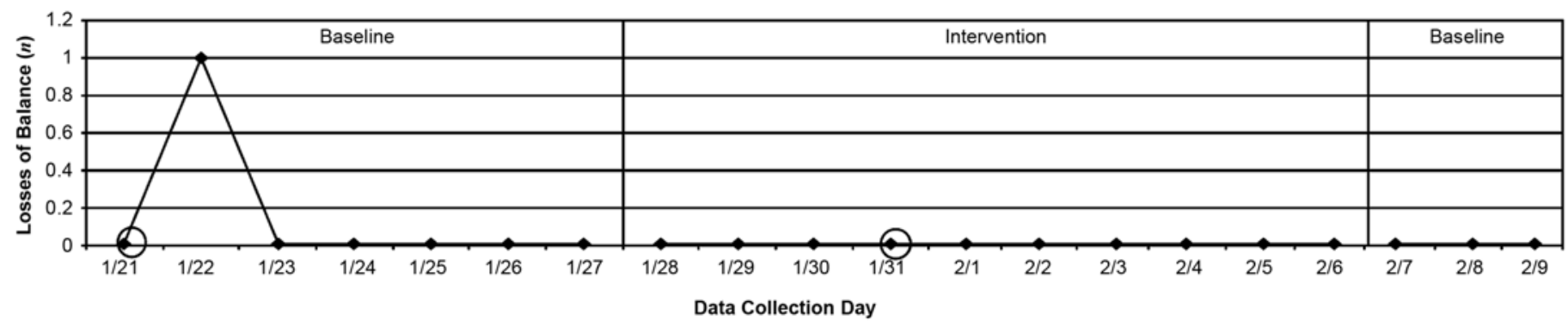

Figure 4.

Daily recording of losses of balance for (a) Participant I, (b) Participant II, and (c) Participant III in applied settings.

changes within each category were variable, with Participant V worsening in all PDQ-39 categories except mobility and support and Participants III and VI improving in all categories. The three other participants had variable declines and improvements.

During the exit interview, all six participants stated that they felt their mobility was improved with use of the VCS. Four participants said that they could initiate walking more easily. Three felt they froze fewer times during the day and were able to move out of a freezing episode more quickly. Four felt that the VCS were more helpful in the community than inside their homes. Two felt the VCS were equally helpful in their homes and in the community. Three participants mentioned that the VCS were especially helpful in doorways and narrow hallways. One stated that he felt his walking speed was improved with the VCS, and an additional participant felt he took longer steps with the VCS. Another participant talked about using the VCS in a proactive manner, tilting his head to start the display if he was entering a situation in which he typically had difficulties. He said that this made him feel more confident, because he "knew the lines would be there if needed." One participant called the investigator 2 months after completing the study and said, "I really had a bad time after I had to give [the VCS] back and wished I had them again . . . I feel they're a good deal.” These comments indicate that the participants felt that the VCS helped their functional mobility. 


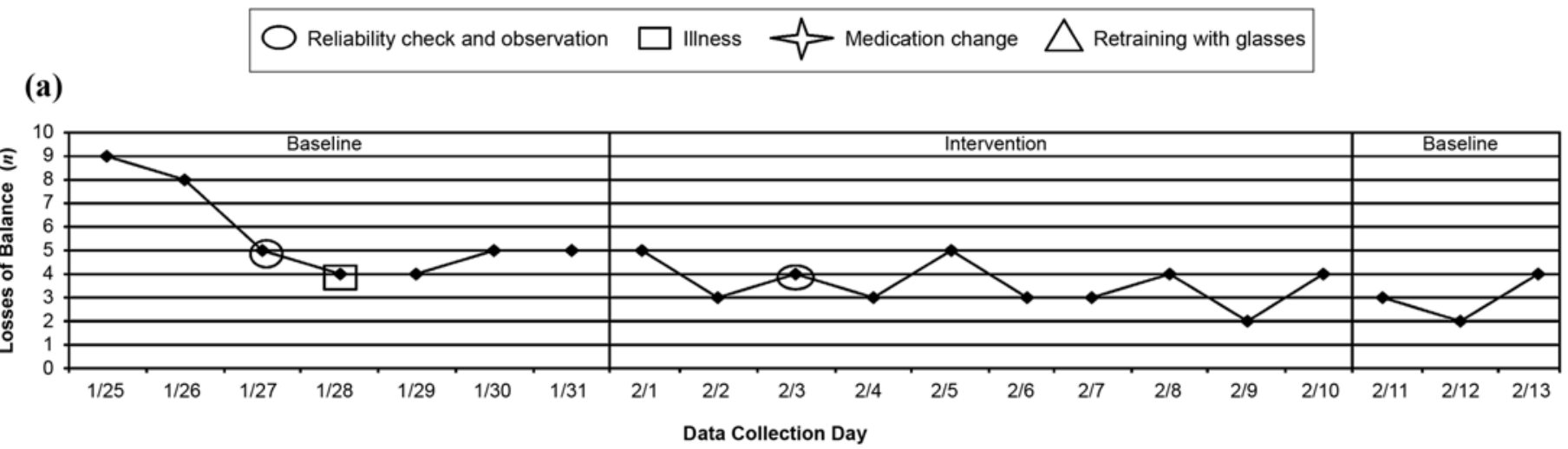

(b)

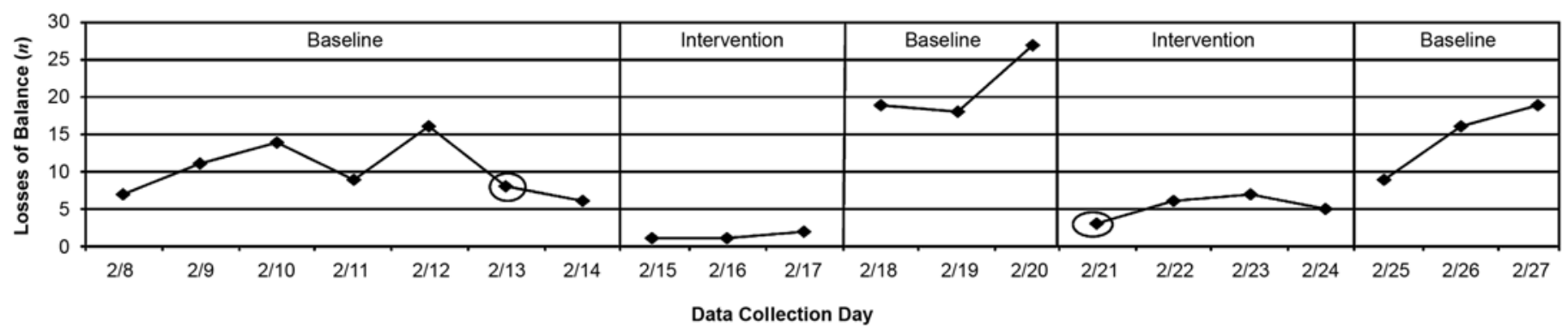

(c)

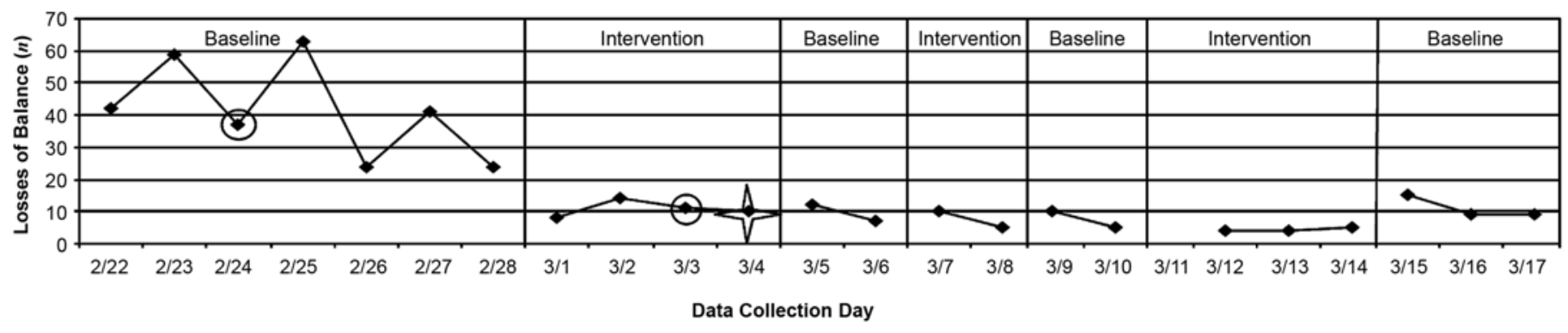

Figure 5.

Daily recording of losses of balance for (a) Participant IV, (b) Participant V, and (c) Participant VI in applied settings.

\section{DISCUSSION}

VCS show promise in simulating kinesia paradoxa with virtual cues in nonclinical home and community settings. Most participants using VCS spent less time in freezes and may have experienced fewer freezes. All participants reported finding VCS helpful.

Results demonstrate that kinesia paradoxa may now be a viable community-based treatment for akinesia through the use of VCS. Virtual cues provide a way for visual cueing to be portable, allowing people with akinesia to take the cues needed for effective mobility into many settings. This is important because to date no nonpharmacologically effective and functional way to treat such individuals has existed, especially in the community.

\section{Limitations}

Because this study was conducted over several weeks, extraneous factors such as medication changes and illness could not be adequately controlled. The design attempted to control for such factors through lengthy baseline and intervention phases of approximately 7 to 10 days per phase. Nevertheless, lifestyle factors are difficult to control in community-based studies. 
The participants chosen were all highly active people, and as a result, they may not have had the difficulties with social isolation and sedentary lifestyle that can be a problem for others in this population. As a result, the makeup of this participant sample limits generalization to people with more severe activity and participation challenges due to PD. While the greatest benefit was observed in the participant with more severe motor difficulty (Participant V), further application of the device with more severely disabled individuals with PD is needed to assess differential effects.

Finally, the same investigator completed all observations. Reliability checks between the participants and investigator indicated good agreement (85.7\%-100.0\%); however, the investigator alone calculated the length of each freeze and reliability checks of these data were not conducted. As a result, ruling out error with this measure is impossible. In addition, only the investigator made the observations about changes in quality of gait, although participant self-reports most often supported those observations.

\section{Future Research}

Replication of this study with more participants with different levels of physical and cognitive disability due to PD would be beneficial. In addition, a lengthier study would help answer questions about the long-term efficacy of VCS. An alternative form of data collection, besides use of daily activity diaries, would also be beneficial. Wearable technology that can measure different types of movement for people with PD is currently being researched [32]. This technology would help provide an objective and accurate picture of the activity and gait patterns of people with PD.

Improvements in VCS would also be helpful. Since the test device was a prototype and improvements are planned, including reducing the overall size and weight, completion of further studies with future models is necessary.

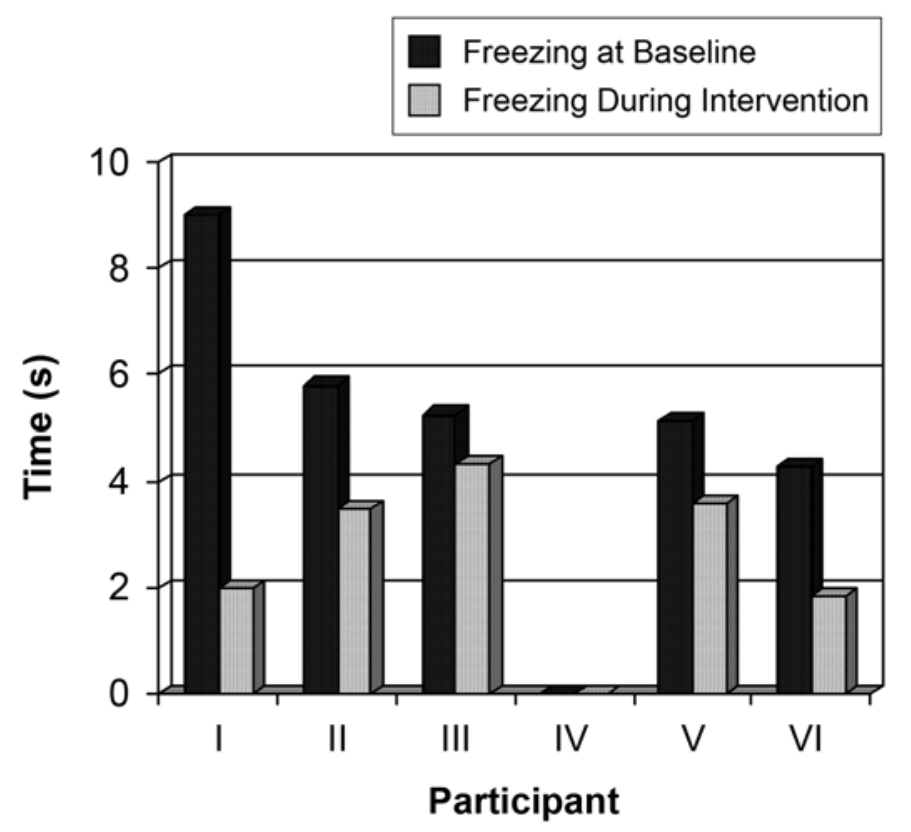

Figure 6.

Average time spent in freeze during observation sessions for all participants. Note: Participant IV was not observed to freeze in either baseline or intervention phases.

Table 2.

Parkinson's Disease Questionnaire-39 scores.

\begin{tabular}{|c|c|c|c|c|c|c|c|c|c|c|c|c|}
\hline \multirow{2}{*}{ Category } & \multicolumn{2}{|c|}{ Participant I } & \multicolumn{2}{|c|}{ Participant II } & \multicolumn{2}{|c|}{ Participant III } & \multicolumn{2}{|c|}{ Participant IV } & \multicolumn{2}{|c|}{ Participant V } & \multicolumn{2}{|c|}{ Participant VI } \\
\hline & Prestudy & Poststudy & Prestudy & Poststudy & Prestudy & Poststudy & Prestudy & Poststudy & Prestudy & Poststudy & Prestudy & Poststudy \\
\hline Mobility & 70.0 & 32.5 & 37.5 & 45.0 & 35.0 & 32.5 & 20.0 & 17.5 & 40.0 & 37.5 & 40.0 & 25.0 \\
\hline Emotion & 20.8 & 25.0 & 45.8 & 29.2 & 8.3 & 8.3 & 8.3 & 12.5 & 0.0 & 0.0 & 37.5 & 33.3 \\
\hline Support & 0.0 & 0.0 & 8.3 & 25.0 & 8.3 & 0.0 & 0.0 & 0.0 & 0.0 & 0.0 & 50.0 & 12.5 \\
\hline Cognition & 50.0 & 43.7 & 25.0 & 18.75 & 12.5 & 0.0 & 6.25 & 12.5 & 37.5 & 50.0 & 12.5 & 12.5 \\
\hline Communication & 16.7 & 66.7 & 25.0 & 25.0 & 16.7 & 16.7 & 41.7 & 66.7 & 41.7 & 50.0 & 50.0 & 41.7 \\
\hline Discomfort & 33.3 & 16.7 & 50.0 & 50.0 & 8.3 & 0.0 & 16.7 & 50.0 & 8.3 & 0.0 & 50.0 & 41.7 \\
\hline
\end{tabular}




\section{CONCLUSIONS}

Effective treatment of akinesia is essential to help people with PD more fully participate in desired activities by managing limitations with functional mobility. This study examined the effects of VCS, a device designed to simulate kinesia paradoxa in homes and communities. Results were encouraging because VCS appeared to improve the functional mobility of all six participants in some way. Future research with new models of VCS is clearly indicated.

\section{ACKNOWLEDGMENTS}

We would like to acknowledge the invaluable contribution of Thomas Riess, inventor of the virtual cueing device used in this research.

This material was based on work supported by a Small Business Innovative Research grant from the National Institutes of Health to HMD Therapeutics (San Anselmo, California), grant 1-R43-NS36503-01A1, and by a summer fellowship to the lead author by the Parkinson's Disease Foundation.

The authors have declared that no competing interests exist.

\section{REFERENCES}

1. Gray P, Hildebrand K. Fall risk factors in Parkinson's disease. J Neurosci Nurs. 2000;32(4):222-28. [PMID: 10994536]

2. Morris ME. Movement disorders in people with Parkinson disease: A model for physical therapy. Phys Ther. 2000; 80(6):578-97. [PMID: 10842411]

3. PADRECC Home [homepage on the Internet]. Philadelphia (PA): U.S. Department of Veterans Affairs; c2001-07 [updated 2006 Nov 8; cited 2006 Aug 17]. Revolutionizing Parkinson's disease care for our veterans; [1 screen]. Available from: http://www.parkinsons.va.gov/index.asp.

4. Giladi N, McMahon D, Przedborski S, Flaster E, Guillory S, Kostic V, Fahn S. Motor blocks in Parkinson's disease. Neurology. 1992;42(2):333-39. [PMID: 1736161]

5. Giladi N, McDermott MP, Fahn S, Przedborski S, Jankovic J, Stern M, Tanner C; Parkinson Study Group. Freezing of gait in PD: Prospective assessment in the DATATOP cohort. Neurology. 2001;56(12):1712-21. [PMID: 11425939]

6. Lamberti P, Armenise S, Castaldo V, De Mari M, Iliceto G, Tronci P, Serlenga L. Freezing gait in Parkinson's disease. Eur Neurol. 1997;38(4):297-301. [PMID: 9434089]
7. Lewis GN, Byblow WD, Walt SE. Stride length regulation in Parkinson's disease: The use of extrinsic, visual cues. Brain. 2000;123(Pt 10):2077-90. [PMID: 11004125]

8. Weghorst S. Augmented reality and Parkinson's disease. Commun ACM. 1997;40(8):47-48.

9. Dietz MA, Goetz CG, Stebbins GT. Evaluation of a modified inverted walking stick as a treatment for Parkinsonian freezing episodes. Mov Disord. 1990;5(3):243-47.

[PMID: 2388642]

10. Morris ME, Iansek R, Matyas TA, Summers JJ. Stride length regulation in Parkinson's disease. Normalization strategies and underlying mechanisms. Brain. 1996;119(Pt 2):551-68. [PMID: 8800948]

11. Fukunaga H, Kasai T, Yoshidome H. Clinical findings, status of care, comprehensive quality of life, daily life therapy and treatment at home in patients with Parkinson's disease. Eur Neurol. 1997;38 Suppl 2:64-69. [PMID: 9387806]

12. Schrag A, Jahanshahi M, Quinn NP. What contributes to depression in Parkinson's disease? Psychol Med. 2001;31(1): 65-73. [PMID: 11200961]

13. Karlsen KH, Tandberg E, Arsland D, Larsen JP. Health related quality of life in Parkinson's disease: A prospective longitudinal study. J Neurol Neurosurg Psychiatry. 2000; 69(5):584-89. [PMID: 11032608]

14. Defebvre L, Blatt JL, Blond S, Bourriez JL, Gieu JD, Destee A. Effect of thalamic stimulation on gait in Parkinson disease. Arch Neurol. 1996;53(9):898-903.

[PMID: 8815855]

15. Stern GM, Lander CM, Lees AJ. Akinetic freezing and trick movements in Parkinson's disease. J Neural Transm Suppl. 1980;(16):137-41. [PMID: 6933218]

16. Thaut MH, McIntosh GC, Rice RR, Miller RA, Rathbun J, Brault JM. Rhythmic auditory stimulation in gait training for Parkinson's disease patients. Mov Disord. 1996;11(2): 193-200. [PMID: 8684391$]$

17. Freedland RL, Festa C, Sealy M, McBean A, Elghazaly P, Capan A, Brozycki L, Nelson AJ, Rothman J. The effects of pulsed auditory stimulation on various gait measurements in persons with Parkinson's disease. NeuroRehabilitation. 2002;17(1):81-87. [PMID: 12016350]

18. Cubo E, Leurgans S, Goetz CG. Short-term and practice effects of metronome pacing in Parkinson's disease patients with gain freezing while in the 'on' state: Randomized single blind evaluation. Parkinsonism Relat Disord. 2004;10(8):507-10. [PMID: 15542012]

19. Suteerawattananon M, Morris GS, Etnyre BR, Jankovic J, Protas EJ. Effects of visual and auditory cues on gait in individuals with Parkinson's disease. J Neurol Sci. 2004; 219(1-2):63-69. [PMID: 15050439]

20. Jiang Y, Norman KE. Effects of visual and auditory cues on gait initiation in people with Parkinson's disease. Clin Rehabil. 2006;20(1):36-45. [PMID: 16502748] 
21. Kompoliti K, Goetz CG, Leurgans S, Morrissey M, Siegel IM. "On” freezing in Parkinson's disease: Resistance to visual cue walking devices. Mov Disord. 2000;15(2):309-12. [PMID: 10752582]

22. Sidaway B, Anderson J, Danielson G, Martin L, Smith G. Effects of long-term gait training using visual cues in an individual with Parkinson disease. Phys Ther. 2006;86(2): 186-94. [PMID: 16445332]

23. Riess T, Weghorst S. Augmented reality in the treatment of Parkinson's disease. In: Morgan K, Satava RM, Sieburg $\mathrm{HB}$, Mattheus R, Christensen JP, editors. Interactive technology and the new paradigm for health care. Seattle (WA): IOS Press; 1995. p. 95-118.

24. Riess T. Gait and Parkinson's disease: A conceptual model for an augmented-reality based therapeutic device. Stud Health Technol Inform. 1998;58:200-208. [PMID: 10350920]

25. Prothero JD. The treatment of akinesia using virtual images [thesis]. Seattle (WA): University of Washington; 1993.

26. Weghorst S, Kaminsky TA. Efficacy of the EVS Parkinson's disease akinesia-aid in first-time users. Seattle (WA): Human Interface Technology Laboratory; 2003.

27. Hoehn MM, Yahr MD. Parkinsonism: Onset, progression and mortality. Neurology. 1967;17(5):427-42. [PMID: 6067254]

28. Goetz CG, Pooewe W, Rascol O, Sampaio C, Stebbins GT, Counsell C, Giladi N, Holloway RG, Moore CG, Wenning
GK, Yahr MD, Seidl L, Movement Disorder Society Task Force on Rating Scales for Parkinson's Disease. Movement Disorder Society Task Force report on the Hoehn and Yahr staging scale: Status and recommendations. Mov Disord. 2004;19(9):1020-28. [PMID: 15372591]

29. Folstein MF, Folstein SE, McHugh PR. "Mini-mental state”: A practical method for grading the cognitive state of patients for the clinician. J Psychiatr Res. 1975;12(3):189-98. [PMID: 1202204

30. Jenkinson C, Fitzpatrick R, Peto V. The Parkinson's disease questionnaire: User manual for the PDQ-39, PDQ-8, and PDQ Summary Index. Oxford (England): Health Services Units, University of Oxford; 1998.

31. Kazdin AE. Single-case research designs: Methods for clinical and applied settings. New York (NY): Oxford University Press; 1982.

32. Van Someren EJ, Vonk BF, Thijssen WA, Speelman JD, Schuurman PR, Mirmiran M, Swaab DF. A new actigraph for long-term registration of the duration and intensity of tremor and movement. IEEE Trans Biomed Eng. 1998; 45(3):386-95. [PMID: 9509754]

Submitted for publication September 5, 2006. Accepted in revised form February 12, 2007. 